\title{
Low voltage electrical activity preceding right atrial depolarisation in man
}

\author{
ALAN F. MACKINTOSH ${ }^{1}$, MICHAEL J. ENGLISH, RICHARD VINCENT, \\ DAVID J. WOOLLONS, AND DOUGLAS A. CHAMBERLAIN
}

From the University of Sussex and the Departments of Cardiology at the Royal Sussex County Hospital, Brighton, and King's College Hospital, London

SUMMARY Electrical recordings were made in the high right atrium in 28 patients undergoing cardiac catheterisation and in 3 healthy volunteers. After filtering and amplification by 3 to 10 million times, the signals were passed through a signal averaging process in a digital computer. Of the 28 subjects who had technically satisfactory recordings, 23 showed low voltage electrical activity preceding the conventionally-recorded atrial depolarisation. The low voltage activity started 50 to $200 \mathrm{~ms}$ before the atrial deflection and was variable in shape. These early signals may be the result of activity in the region of the sinus node.

The rhythm of the healthy heart is controlled by the depolarisation of pacemaker cells in the sinus node. Animal sinus node tissue can be suspended in an organ bath and intracellular and extracellular recordings made from the pacemaker cells (West, 1955; Cramer et al., 1977), but few convincing recordings have been made of the functioning sinus node in an intact mammalian heart. Some tracings have shown early activity in the high right atrium (Van der Kooi et al., 1956; Ramlau, 1974; Théry et al., 1976) but these early, high frequency signals are not clearly distinct from the earliest atrial activity (Masuda and Paes de Carvalho, 1975). No verified demonstrations have been published of the low frequency, low amplitude signals which would be expected to precede atrial activity in the high right atrium (Cramer et al., 1977).

Hoffman and Cranefield (1960) predicted that sinus node activity should be recordable by small electrodes relatively far apart. The fundamental problem is that the very small signals may be smaller than the random background electrical activity ('noise'). In such circumstances an increase in amplification would not make the physiological signals any more discernible. We have attempted to record activity in the high right atrium in man using high amplification combined with signal averaging to reduce random background noise.

'Present address: Regional Cardiac Unit, Papworth Hospital, Cambridge.

Received for publication 4 April 1979.

\section{Method}

Recordings were made with commercially-available hexapolar pacing wires using two electrodes 3.5 to $10 \mathrm{~cm}$ apart (Berkowitz-Castellanos, USCI). The pacing wire was inserted via a femoral, subclavian, or antecubital vein and positioned under fluoroscopic control with one electrode near the superior vena cava/right atrial junction and the other recording electrode in the superior vena cava or its tributaries.

The bipolar signals were amplified a thousand times with a low-noise, battery-powered amplifier (Ancom Limited, B9A-7D) and recorded on one channel of a four-channel tape recorder (Racal Thermionics, Store 4). This low-gain channel was used as a trigger for the signal averaging process (Fig. 1). After this initial amplification the same signals were filtered with a high pass cut-off of 3 to $10 \mathrm{~Hz}$ (Barr and Stroud EF3 filter, damped setting), amplified further by 10 to 100 times and recorded on another channel of the tape recorder. Lead I or II was recorded simultaneously.

On replay, in the computing laboratory, the high gain intra-atrial signals were filtered with a low pass cut-off of $150 \mathrm{~Hz}$ and a variable high pass cutoff within the range 10 to $20 \mathrm{~Hz}$. At settings below $10 \mathrm{~Hz}$ the DC drift was too great to permit a high degree of amplification. With a cut-off above $20 \mathrm{~Hz}$ the signals of interest became less prominent. The high pass filtering was adjusted to remove as much as possible of the preceding $T$ or $U$ wave. The best 


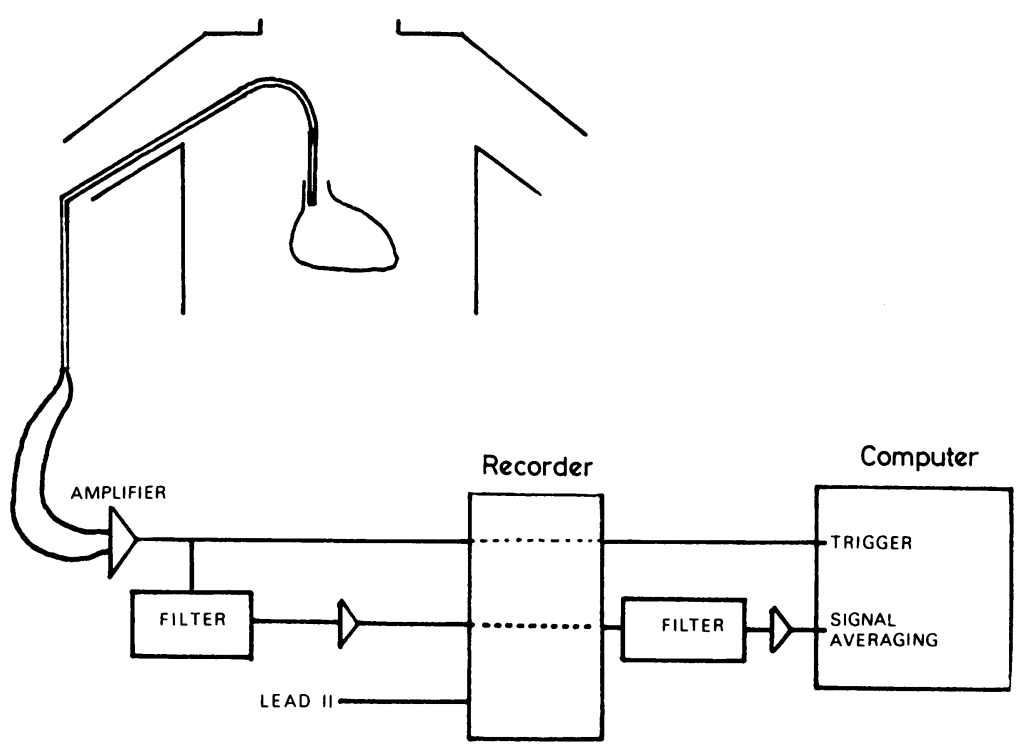

Fig. 1 Block diagram of the recording and processing system.

setting was usually between 10 and $14 \mathrm{~Hz}$. The filtered, high-gain signals were amplified again to produce a total gain of 3 to 10 million.

For signal averaging, the cycles were added together in a general-purpose digital computer (Computer Technology Limited, Modular One). The analogue to digital converter accepted signal voltages in the range $\pm 5 \mathrm{~V}$ with a resolution of $5 \mathrm{mV}$ per bit. The sampling rate was 3500 to $4000 \mathrm{~Hz}$. The atrial depolarisation of the low gain channel was used to align the cycles correctly. Sixty to 600 cycles were averaged depending on the ease with which the noise was reduced, though at the high degree of amplification it was impossible to remove the random noise entirely. The results were plotted on a digital X-Y plotter (Calcomp 565). If necessary residual high frequency noise could be reduced by digital filtering before plotting.

The system of amplification and signal averaging was originally developed for the recording of external His bundle potentials. An earlier account (Vincent et al., 1978) showed that a small artificial signal could be readily extracted from random noise by this signal-averaging system.

Acceptable triggering could be obtained using a simple threshold trigger on the upstroke of the low gain deflection, but results were more satisfactory with a pattern matching technique. For this technique the waveform of each atrial depolarisation was compared with a stored atrial signal. The beginning of the fresh signal was adjusted in time until the computer found the position for the best match with the stored signal. If the match was not close enough, that cycle was discarded. The beginning was then used as a reference point to add in the high gain signal to the averaging process.

As the exact position of the sinus node cannot be determined by fluoroscopy, simultaneous recordings were usually made with two pairs of electrodes on a single multipolar pacing wire. The active poles in the high right atrium were $1 \mathrm{~cm}$ apart.

\section{Subjects}

Recordings have been made on 3 healthy volunteers and 28 patients undergoing routine diagnostic cardiac catheterisation (Table). We have tended to select patients with slower heart rates, who have some separation between the electrical activity of the $T$ wave and that of the following atrial depolarisation.

\section{Results}

Of the 31 subjects, 23 had recognisable electrical activity preceding the conventionally-recorded atrial activity in at least one recording. An example

\section{Table Diagnosis in 31 subjects}

\begin{tabular}{ll}
\hline Diagnosis & No \\
\hline Sinus node disease & 9 \\
Other conducting system disease & 8 \\
Ischaemic heart disease & 7 \\
Normal volunteers & 3 \\
Miscellaneous & 4 \\
\hline
\end{tabular}


is shown in Fig. 2. Of the remaining 8 subjects, 5 had no clear interval between the end of the $T$ or $U$ wave and the start of atrial activity, 2 had unsatisfactory trigger signals, and the remaining 1 had distinct mains interference, which could not be removed by signal averaging.

The morphology of the early signal was variable. The commonest pattern (18 out of 23) showed an ill-defined onset with the early signal continuing into the atrial activity (Fig. 3 and 4). Four subjects showed little activity between the early signal and atrial depolarisation (Fig. 5). One subject with sinus node disease had a short, early signal with an abrupt onset and then a gap before the atrial deflection (Fig. 6).

The time from the onset of the early high gain signal to the start of the atrial activity in the low
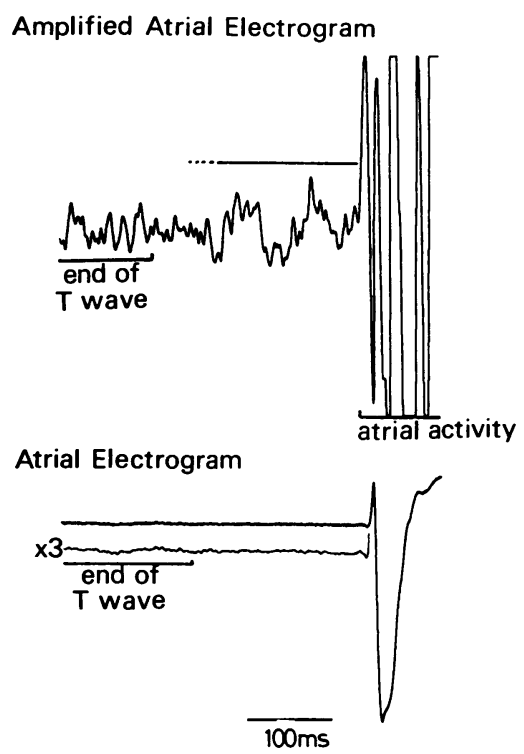

Lead II

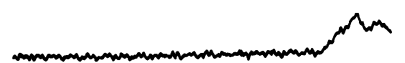

Fig. 2 Simultaneous display of the highly-amplified atrial signals after the signal averaging process (top), the conventionally-recorded atrial electrogram (middle), and the electrocardiogram from the end of the $T$ wave to the $P$ wave (bottom). The horizontal line marks the approximate extent of the early signal. Part of the atrial electrogram is shown amplified 3 more times. The atrial activity in the highly-amplified recording is saturated. The filtering of the highly-amplified signal has reduced the duration of the $T$ wave. gain electrogram was in the region of 50 to $200 \mathrm{~ms}$. In all cases the internal atrial activity was compared with the external $\mathbf{P}$ wave recorded in at least 2 (and usually 3) planes at right-angles to each other. The external signals never began before the internal activity. In this small series we found no systematic difference in the time from the onset of the early signal to the start of atrial activity between healthy volunteers and patients with sinus node disease. The longest interval (just under $200 \mathrm{~ms}$ ) was in a 29-year-old man with syncope but no proven heart disease. The second longest (about $160 \mathrm{~ms}$ ) was in a healthy 30 -year-old volunteer.

The shape and, to a lesser extent, the time of onset of the early signal is dependent on the degree of high pass filtering used. It is, therefore, impossible to measure precisely the peak amplitude of the early signal but it may be in the range of $200-500$ nanovolts.

The early signal was reproducible if a recording was reanalysed. In Fig. 7 and 8, the recordings of 2

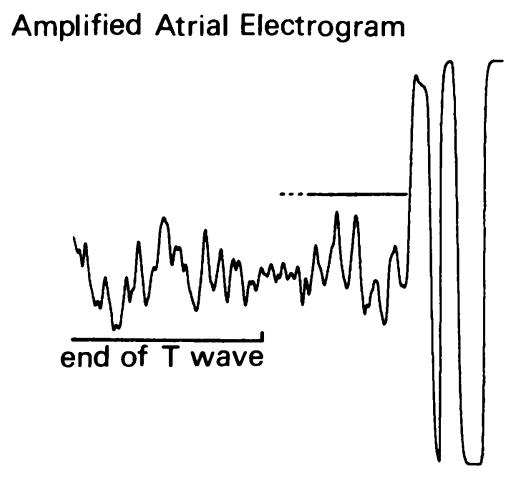

Atrial Electrogram

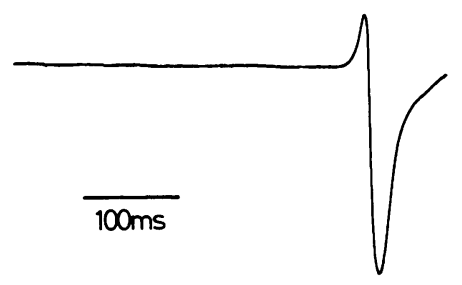

Lead II

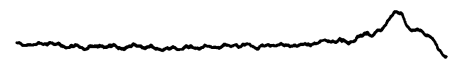

Fig. 3 Another simultaneous display of the atrial signals after the averaging process, the atrial electrogram and the electrocardiogram. The horizontal line marks the approximate extent of the early signal. 
further subjects were reprocessed using nonidentical cycles for the averaging technique. Some variations have occurred in the shapes of the early signals but the timings are unchanged.

\section{Discussion}

Sinus node disease is an important cause of morbidity and possible mortality, yet we do not know the aetiology in most cases (Shaw, 1976). Existing methods of diagnosis are unsatisfactory. If the electrocardiogram is normal, the most useful diagnostic procedure is 24 -hour ambulatory monitoring (Lipski et al., 1976). This technique reveals little or nothing of underlying mechanisms. The recovery time of the sinus node after overdrive suppression (Mandel et al., 1971) and attempted calculations of the sinuatrial conduction time
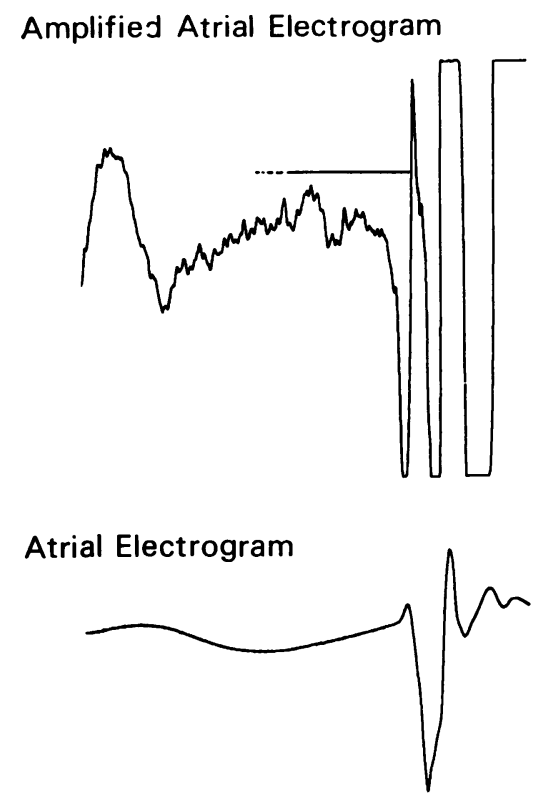

$\overline{100 \mathrm{~ms}}$

Lead II

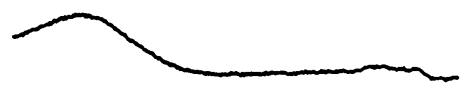

Fig. 4 An example of the commonest pattern for the early signal. After an ill-defined onset the signal continues into the atrial activity.
(Strauss et al., 1973) have proved to be of little diagnostic value in most cases (Crook et al., 1977). Any method which reveals direct information about the human sinus node would increase our understanding of sinus node disease and may ultimately have diagnostic implications.

We have demonstrated early signals preceding atrial depolarisation compatible with activity in, or close to, the sinus node. Their character and timing are similar to those predicted for the human sinus node signal, but there is no direct method of proving their origin. Previous recordings with epicardial electrodes placed on the sinus node of experimental animals have shown high frequency activity starting about $30 \mathrm{~ms}$ before the conventionally recorded atrial signal (Van der Kooi et al., 1956; Ramlau, 1974; Théry et al., 1976). But this

\section{Amplified Atrial Electrogram}

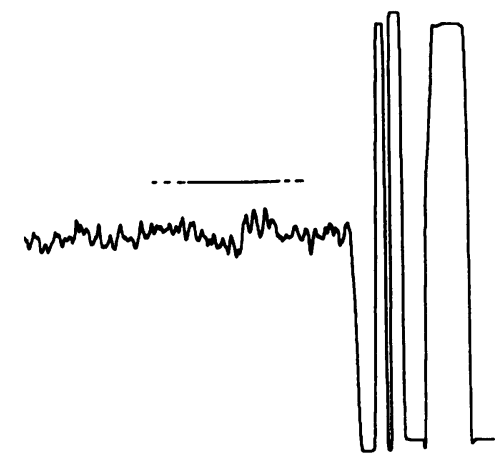

Atrial Electrogram

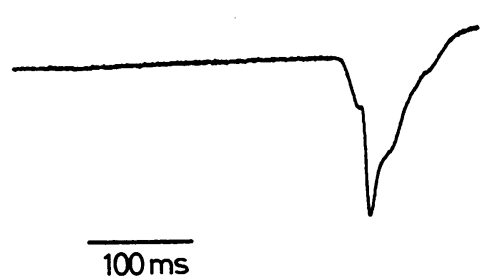

Lead II

Fig.'5 Another pattern for the early signal; the activity appears to diminish before the atrial depolarisation. 
high frequency activity is more likely to be produced by atrial rather than sinus node depolarisation (Masuda and Paes de Carvalho, 1975). CastilloFenoy et al. (1977) discovered early low frequency signals with a unipolar electrode applied to the dog sinus node. This presumed sinus node activity was recorded with surprising ease but clamps had been applied to the atria and the dog rendered anoxic to obtain the signals. Using signal averaging, Stopczyk et al. (1979) recorded early activity with intraatrial electrodes in both dogs and humans, but these signals are not distinct from atrial activity or the end of ventricular repolarisation in the previous cycle. Similar studies in humans have been briefly reported, without illustrations or technical details, by other workers (Krongrad et al., 1978; Tomita et al., 1978). Cramer et al. (1977) have described extracellular potentials recorded from an isolated rabbit sinus node preparation. There were two

\section{Amplified Atrial Electrogram}

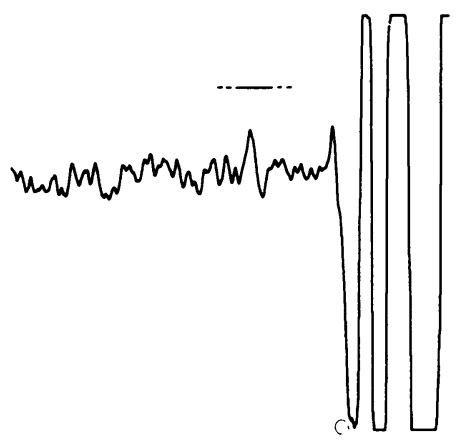

Atrial Electrogram

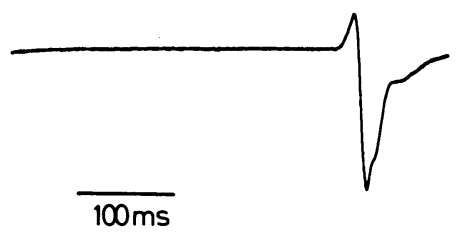

Lead II

Fig. 6 In this patient with sinus node disease the early signal has an abrupt onset with a gap before the atrial depolarisation. potential changes. One was a slow continuous diastolic depolarisation; with our technique this would be removed by filtering. The other potential change was a faster depolarisation which began about $70 \mathrm{~ms}$ before the atrial activity. After filtering this second depolarisation would be similar in shape and timing to our early signals.

The time from the onset of our early signals to the earliest atrial activity was up to $200 \mathrm{~ms}$. In the isolated rabbit atrial preparation the sinuatrial conduction time is 25 to $90 \mathrm{~ms}$ (Miller and Strauss, 1974; Yamaguchi and Mandel, 1975). In the slower human heart the sinuatrial conduction time is likely to be longer. In our experiments the apparent onset of atrial activity may have been influenced by the plane of the recording electrodes. However, any error must have been small because the internal atrial activity always began simultaneously with, or only just before, the external $P$ wave recorded in at least two orthogonal planes.
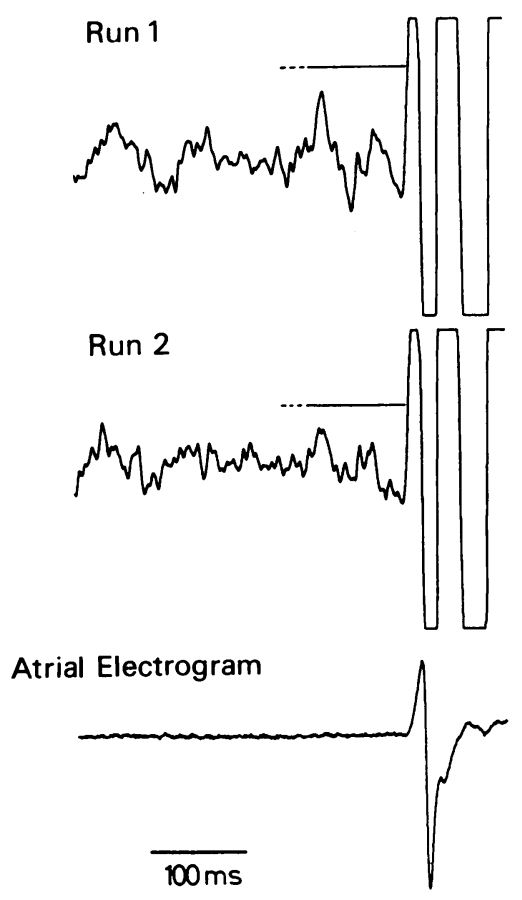

Lead II

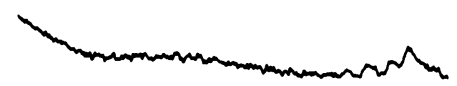

Fig. 7 A comparison of the effects of averaging non-identical cycles from the same recording. The early signal varies in shape but not in timing. 
The early signal was reproducible in the same patient if another set of cycles was used for the averaging process. Unfortunately not all patients were able to lie still for sufficient time to record enough cycles. The results from 2 subjects who were able to remain quiet are shown in Fig. 7 and 8. The timing of the early signal has not changed appreciably but the shape has altered. Bonke et al. (1971) showed that the pacemaker site within the sinus node of rabbits shifted after premature atrial stimulation, and indirect evidence suggested that the point of impulse formation also varied during sinus rhythm in man (Cabanis et al., 1978). Variations in shape of the early signal might be due to changes in the depolarisation wave within the sinus node.

We had to overcome two problems relating to the position of our electrodes. First, difficulty over the uncertain location of the sinus node was reduced by making simultaneous recordings from the active

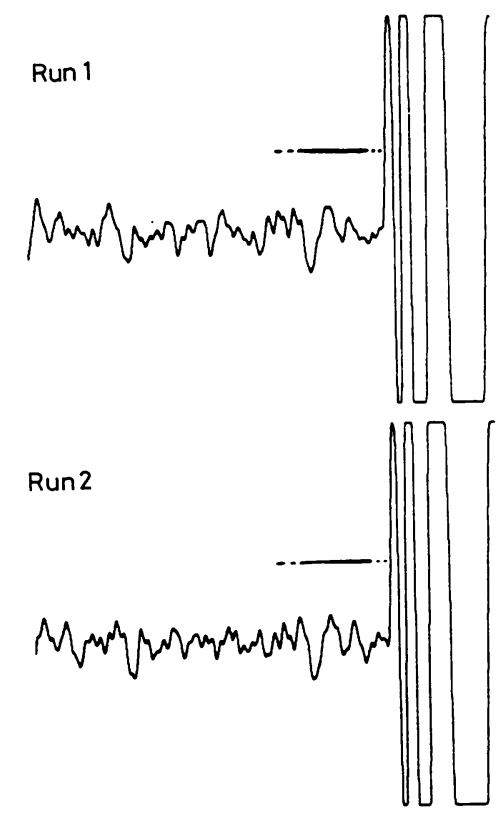

Atrial electrogram

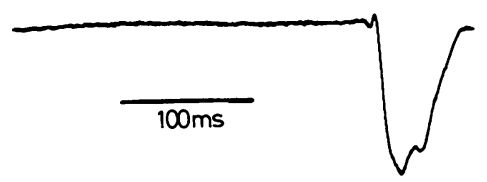

Fig. 8 Another example of the effects of averaging non-identical cycles from the same recording. Again the early signal varies in shape but not in timing. poles of two pairs of electrodes placed slightly differently in the upper right atrium; the better result was selected. Secondly, the effects of respiratory movement were reduced by the adoption of a pattern-matching technique for triggering. By accepting only a close match, signal averaging can be confined to signals sensed when the distal electrode is within a small area in the high right atrium. The pattern matching will also eliminate any ectopic beats from thr averaging process, as ectopic or retrograde $P$ waves will vary in shape from sinus ones.

Possible sources other than sinus node depolarisation should be considered for these early signals. The signals are likely to represent electrical activity within the heart; any signals which are not related to the cardiac cycle should be eliminated by the signal averaging. Previous recordings of early activity beginning 20 to $30 \mathrm{~ms}$ before the conventionally-recorded high right atrial activity have probably been produced by the onset of atrial depolarisation. Our early signals often begin sooner, over $100 \mathrm{~ms}$ before the conventionally-recorded atrial activity; it is unlikely that they are produced by the onset of atrial depolarisation. The end of the $\mathrm{T}$ or $\mathrm{U}$ wave of the previous cycle is a more plausible source for these early signals. A ventricular repolarisation signal may have frequency components similar to those of a sinus node signal, producing comparable waveforms on the recordings. However, any distant ventricular repolarisation would be continuous with the end of the $T$ wave but not necessarily with the atrial depolarisation of the following cycle. When the heart rate was slow enough we observed a gap between the end of the $T$ or $U$ wave and our early signal. Moreover, the early signal continued into the atrial activity in 18 out of 23 cases.

The clinical implications of the recognition of these early signals is uncertain. We have not yet found any consistent difference in the shape or timing between patients with and without sinus node dysfunction, but the numbers are too small to draw firm conclusions. Furthermore, our experimental method may itself distort results especially if patients with sinus node dysfunction have variable conduction times from sinus node to atrium. Since our recording technique depends on the averaging of many cycles, good results can be obtained only when the time from sinus node depolarisation to atrial activation is fairly constant. Thus, subjects with potentially the most interesting results may produce the poorest recordings.

Despite inherent limitations in the present technique, the inevitable difficulty in the interpretation of very low voltage signals, and our uncertainty 
about clinical significance, we believe that studies of this type should be continued because sinus node activity is both important and ill-understood. Knowledge of sinus node function will be enhanced by reliable recordings of the electrical activity generated within it.

This work was carried out with the aid of grants from the British Heart Foundation. We are also indebted to Dr Oram and Dr Jewitt for their cooperation in this project.

\section{References}

Bonke, F. I. M., Bouman, L. N., and Schopman, F. J. G. (1971). Effect of an early atrial premature beat on activity of the sino-atrial node and atrial rhythm in the rabbit. Circulation Research, 29, 704-715.

Cabanis, C., Guize, L., Le Heuzey, J. Y., Pauly, N., Zacouto, F., and Maurice, P. (1978). Évaluation de la fonction sinoauriculaire par la méthode des extrasystoles provoquées de prématurité relative constante. I. Méthode et resultats normaux. Archives des Maladies du Coeur et des Vaisseaux, 71, 1113-1120.

Castillo-Fenoy, A., Valère, P. E., Guérot, C., and Tricot, R. (1977). Enregistrement de l'activité électrique de la région sinusale au cours d'un bloc sino-auriculaire expérimental chez le chien. Arguments en faveur de son origine sinusale. Archives des Maladies du Coeur et des Vaisseaux, 70, 273-278.

Cramer, M., Siegal, M., Bigger, J. T., jun, and Hoffman, B. F. (1977). Characteristics of extracellular potentials recorded from the sinoatrial pacemaker of the rabbit. Circulation Research, 41, 292-300.

Crook, B., Kitson, D., McComish, M., and Jewitt, D. (1977). Indirect measurement of sinoatrial conduction time in patients with sinoatrial disease and in controls. British Heart fournal, 39, 771-777.

Hoffman, B., and Cranefield, P. (1960). Electrophysiology of the Heart. McGraw Hill, New York.

Krongrad, E., Hariman, R. J., Boxer, R. A., Weiss, M. B., Steeg, C. N., and Hoffman, B. F. (1978). A method for recording of sinus node and ectopic atrial pacemaker electrograms during cardiac catheterization in human (abstract). Circulation, 58, Suppl. 11, 146.

Lipski, J., Cohen, L., Espinoza, J., Motro, M., Dack, S., and Donoso, E. (1976). Value of Holter monitoring in assessing cardiac arrhythmias in symptomatic patients. American fournal of Cardiology, 37, 102-107.
Mandel, W., Hayakawa, H., Danzig, R., and Marcus, H. S. (1971). Evaluation of sinoatrial node function in man by overdrive suppression. Circulation, 44, 59-66.

Masuda, M. O., and Paes de Carvalho, A. (1975). Sinoatrial transmission and atrial invasion during normal rhythm in the rabbit heart. Circulation Research, 37, 414-421.

Miller, H. C., and Strauss, H. C. (1974). Measurement of sinoatrial conduction time by premature atrial stimulation in the rabbit. Circulation Research, 35, 935-947.

Ramlau, R. A. (1974). Electrograms of the sinuatrial node in dogs following surgical implantation of electrodes on the epicardium. Fournal of Electrocardiology, 7, 137-148.

Shaw, D. B. (1976). The etiology of sino-atrial disorder (sick sinus syndrome). American Heart fournal, 92, 539-540.

Stopczyk, M. J., Wajszczuk, W. J., Zochowski, R. J., and Rubenfire, M. (1979). Pre-P (sino-atrial nude region) activity recording from the right atrial cavity by signal averaging. Pace, 2, 156-161.

Strauss, H. C., Saroff, A. L., Bigger, J. T., and Giardina, E. G. (1973). Premature atrial stimulation as a key to the understanding of sino-atrial conduction in man. Circulation, 47, 86-93.

Théry, C., Lekieffre, J., Lemaire, P., Asseman, P., Dup'is, B., and Warembourg, H. (1976). L'activité électrique de la région du noeud sinusal. Étude experimentale. Archives des Maladies du Coeur et des Vaisseaux, 69, 661-669.

Tomita, T., Matsumoto, Y., Kodama, H., Hashimoto, H., Miyata, K., Yaginuma, T., and Hosoda, S. (1978). Preatrial activity recorded by signal averaging technic of intraatrial electrogram: experience in clinical analysis of sinoatrial conduction (abstract). In Proceedings of the VIII World Congress of Cardiology, p. 449. Tokyo.

Van der Kooi, M. W., Durrer, D., Van Dam, R. T., and Van der Tweel, L. H. (1956). Electrical activity in sinus node and atrioventricular node. American Heart fournal, 51, 684-700.

Vincent, R., Stroud, N. P., Jenner, R., English, M. J., Woollons, D. J., and Chamberlain, D. A. (1978). Noninvasive recording of electrical activity in the PR segment in man. British Heart fournal, 40, 124-130.

West, T. C. (1955). Ultramicroelectrode recording from the cardiac pacemaker. Fournal of Pharmacology and Experimental Therapeutics, 115, 283-290.

Yamaguchi, I., and Mandel, W. (1975). Effects of antiarrhythmic drugs on the sinus node to atrial conduction time in isolated atrial tissue (abstract). Circulation, $\mathbf{5 1}$ and 52, Suppl. II, 259.

Requests for reprints to Dr A. F. Mackintosh, Regional Cardiac Unit, Papworth Hospital, Papworth Everard, Cambridge CB3 8RE. 